\title{
Quantum transport via evanescent waves in undoped graphene
}

\author{
M. I. Katsnelson \\ Radboud University Nijmegen, Institute for Molecules and Materials, \\ Heijendaalseweg 135, 6525 AJ, Nijmegen, The Netherlands
}

\begin{abstract}
Charge carriers in graphene are chiral quasiparticles ("massless Dirac fermions"). Graphene provides therefore an amazing opportunity to study subtle quantum relativistic effects in condensed matter experiment. Here I review a theory of one of these unusual features of graphene, a "pseudodiffusive" transport in the limit of zero charge carrier concentration, which is related to existence of zero-modes of the Dirac operator and to the Zitterbewegung of unltrarelativistic particles. A conformal mapping technique is a powerful mathematical tool to study these phenomena, as demonstrated here, using the Aharonov-Bohm effect in graphene rings with Corbino geometry as an example.
\end{abstract}

Keywords: Graphene, Dirac equation, pseudodiffusive transport, minimal conductivity, Aharonov - Bohm effect, conformal mapping

\section{INTRODUCTION}

Graphene, a recently discovered two-dimensional allotrope of carbon, is a subject of a hot scientific interest (for review, see Refs. 1 7). One of the most unusual features of this new material is a peculiar character of electron energy spectrum: the charge carriers in graphene are chiral quasiparticles described by a two-dimensional analog of massless Dirac equation with effective "velocity of light" of order of $10^{6} \mathrm{~ms}^{-1}$, that is, three hundreds times smaller than for real massless particles 8.9 . This opens a way to study quantum relativistic effects, which were unreachable in high energy physics, by condensed matter experiments. As an example, it is worth to mention the theoretical prediction ${ }^{10}$ and experimental observation $\frac{11,12}{\text { of }}$ the Klein tunneling in graphene.

Another amazing property of graphene is the finite minimal conductivity which is of the order of the conductance quantum $e^{2} / h$ per valley per $\operatorname{spin}^{8}+\frac{9}{.}$. Numerous considerations of the conductivity of a two-dimensional massless Dirac fermion gas do give this value of the minimal conductivity with the accuracy of some factor of order of unity $\underline{13} \underline{\underline{23}}$.

It is really surprising that in the case of massless two-dimensional Dirac fermions there is a finite conductivity for an ideal crystal, that is, without any scattering processes $15,21-23$. This was first noticed in Ref. 15 using a quite complicated formalism of conformal field theory (see also a more detailed and complete discussion in Ref. 23). After a discovery of the minimal conductivity in graphene ${ }^{8.9}$ I was pushed by my colleagues experimentalists to give a more transparent physical explanation of this fact which has been done in Ref. 21, based on the concept of Zitterbewegung ${ }^{24}$ and on the Landauer formula 25,26 . The latter approach has been immediately developed further and used to calculate the shot noise ${ }^{22}$ which turns out to be similar to that in strongly disordered metals (a "pseudodiffusive transport"). There are now more theoretical $27-31$ and experimental ${ }^{32,33}$ works studying this regime in a context of graphene. This situation is very special. For conventional electron gas in semiconductors, in the absence of disorder, the states with definite energy (eigenstates of the Hamiltonian) can be simultaneously the states with definite current (eigenstates of the current operator) and it is the disorder that results in non-conservation of the current and finite conductivity. Contrary, for the Dirac fermions the current operator does not commute with the Hamiltonian ("Zitterbewegung") which can be considered as a kind of intrinsic disorder ${ }^{21,34}$. Therefore, more detailed understanding of the pseudodiffusive transport in graphene is not only important for physics of graphene devices but also has a great general interest for quantum statistical physics and physical kinetics.

\section{ZITTERBEWEGUNG AS AN INTRINSIC DISORDER}

The Zitterbewegung is a quantum relativistic phenomenon which was first discussed by Schrödinger as early as in $1930^{24}$. Only very recently, it was observed experimentally for trapped ions $\frac{35}{3}$. This phenomenon seems to be important to understand qualitatively peculiarities of electron transport in graphene at small doping21,34. Other aspects of the Zitterbewegung in graphene physics, in particular, possibilities of its direct experimental observation, are discussed in Refs $\underline{36} \underline{-38}$. Here we will explain this basic concept for the case of two-dimensional massless Dirac fermions.

We start with the Hamiltonian

$$
H=v \sum_{\mathbf{p}} \Psi_{\mathbf{p}}^{\dagger} \sigma \mathbf{p} \Psi_{\mathbf{p}}
$$


and the corresponding expression for the current operator

$$
\mathbf{j}=e v \sum_{\mathbf{p}} \Psi_{\mathbf{p}}^{\dagger} \sigma \Psi_{\mathbf{p}}=\sum_{\mathbf{p}} \mathbf{j}_{\mathbf{p}}
$$

where $v$ is the electron velocity, $\sigma=\left(\sigma_{x}, \sigma_{y}\right)$ are Pauli matrices, $\mathbf{p}$ is the momentum, and $\Psi_{\mathbf{p}}^{\dagger}=\left(\psi_{\mathbf{p} 1}^{\dagger}, \psi_{\mathbf{p} 2}^{\dagger}\right)$ are pseudospinor electron operators. The expression (21) follows from Eq.(11) and the gauge invariance which requires ${ }^{39}$

$$
\mathbf{j}_{\mathbf{p}}=\frac{\delta H}{\delta \mathbf{p}}
$$

Here we omit spin and valley indices (so, keeping in mind applications to graphene, the results for the conductivity should be multiplied by 4 due to two spin projections and two conical points per Brillouin zone). Straightforward calculations give for the time evolution of the electron operators $\Psi(t)=\exp (i H t) \Psi \exp (-i H t)(\hbar=1)$ :

$$
\Psi_{\mathbf{p}}(t)=\frac{1}{2}\left[e^{-i \epsilon_{\mathbf{p}} t}\left(1+\frac{\mathbf{p} \sigma}{p}\right)+e^{i \epsilon_{\mathbf{p}} t}\left(1-\frac{\mathbf{p} \sigma}{p}\right)\right] \Psi_{\mathbf{p}}
$$

and for the current operator

$$
\begin{aligned}
\mathbf{j}(t) & =\mathbf{j}_{0}(t)+\mathbf{j}_{1}(t)+\mathbf{j}_{1}^{\dagger}(t) \\
\mathbf{j}_{0}(t) & =e v \sum_{\mathbf{p}} \Psi_{\mathbf{p}}^{\dagger} \frac{\mathbf{p}(\mathbf{p} \sigma)}{p^{2}} \Psi_{\mathbf{p}} \\
\mathbf{j}_{1}(t) & =\frac{e v}{2} \sum_{\mathbf{p}} \Psi_{\mathbf{p}}^{\dagger}\left[\sigma-\frac{\mathbf{p}(\mathbf{p} \sigma)}{p^{2}}+\frac{i}{p} \sigma \times \mathbf{p}\right] \Psi_{\mathbf{p}} e^{2 i \epsilon_{\mathbf{p}} t}
\end{aligned}
$$

where $\epsilon_{\mathbf{p}}=v p$ is the particle energy. The last line in Eq.(5) corresponds to the Zitterbewegung.

Its physical interpretation is usually given in terms of Landau-Peierls generalization of the Heisenberg uncertainty principle $\underline{4041}$. Attempts to measure the coordinate of a relativistic particle with a very high accuracy requires the energy which is sufficient to create particle-antiparticle pairs and, thus, we will inevitably loose our initial particle, being not able to distinguish it from one of the created ones (according to quantum statistics, all microparticles are equivalent). This pair creation corresponds to the oscillating terms with the frequency $2 \epsilon_{\mathbf{p}}$ in Eq.(5).

In terms of condensed matter physics, the Zitterbewegung is nothing but a special kind of inter-band transitions with creation of virtual electron-hole pairs. The unitary transformation generated by the operator $U_{\mathbf{p}}=1 / \sqrt{2}\left(1+i \mathbf{m}_{\mathbf{p}} \sigma\right)$, where $\mathbf{m}_{\mathbf{p}}=\left(\cos \phi_{\mathbf{p}},-\sin \phi_{\mathbf{p}}\right)$ and $\phi_{\mathbf{p}}$ is the polar angle of the vector $\mathbf{p}$, diagonalizes the Hamiltonian and thus introduces electron and hole states with the energies $\pm v p$; after this transformation the oscillating term in Eq.(15) corresponds obviously to the inter-band transitions, e.g.

$$
U_{\mathbf{p}}^{\dagger} j_{\mathbf{p}}^{x} U_{\mathbf{p}}=e v\left(\begin{array}{cc}
-\cos \phi_{\mathbf{p}} & -i \sin \phi_{\mathbf{p}} e^{-i \phi_{\mathbf{p}}+2 i \epsilon_{\mathbf{p}} t} \\
i \sin \phi_{\mathbf{p}} e^{i \phi_{\mathbf{p}}-2 i \epsilon_{\mathbf{p}} t} & \cos \phi_{\mathbf{p}}
\end{array}\right)
$$

To calculate the conductivity $\sigma(\omega)$ one can try first to use the Kubo formula ${ }^{42}$ which reads for two-dimensional isotropic case:

$$
\sigma(\omega)=\frac{1}{2 A} \int_{0}^{\infty} d t e^{i \omega t} \int_{0}^{\beta} d \lambda\langle\mathbf{j}(t-i \lambda) \mathbf{j}\rangle
$$

where $\beta=T^{-1}$ is the inverse temperature, $A$ is the sample area. In the static limit $\omega=0$ taking into account Onsager relations and analyticity of the correlators $\langle\mathbf{j}(z) \mathbf{j}\rangle$ for $-\beta<\operatorname{Im} z \leq 0$ one has $\underline{42}$

$$
\sigma=\frac{\beta}{4 A} \int_{-\infty}^{\infty} d t\langle\mathbf{j}(t) \mathbf{j}\rangle
$$

Usually, for ideal crystals, the current operator commutes with the Hamiltonian and thus $\mathbf{j}(t)$ does not depend on time. In that case, due to Eq.(7) the frequency-dependent conductivity contains only the Drude peak

$$
\sigma_{D}(\omega)=\frac{\pi}{2 A} \lim _{T \rightarrow 0} \frac{\left\langle\mathbf{j}^{2}\right\rangle}{T} \delta(\omega)
$$


Either the spectral weight of the Drude peak is finite and, thus, the static conductivity is infinite, or it is equal to zero. It is easy to check that for the system under consideration the spectral weight of the Drude peak is proportional to the modulus of the chemical potential $|\mu|$ and thus vanishes at zero doping $(\mu=0)$. It is the Zitterbewegung, i.e. the oscillating term $\mathbf{j}_{1}(t)$ which is responsible for nontrivial behavior of the conductivity for zero temperature and zero chemical potential. A straightforward calculation gives a formal result

$$
\sigma=\frac{\pi e^{2}}{2 h} \int_{0}^{\infty} d \epsilon \epsilon \delta^{2}(\epsilon)
$$

where one delta-function originates from the integration over $t$ in Eq.(8) and the second one - from the derivative of the Fermi distribution function appearing at the calculation of the average over product of Fermi-operators. Of course, the square of the delta function is not a well-defined object and thus Eq.(10) is meaningless before specification of the way how one should regularize the delta-functions. After regularization the integral in Eq.(10) is finite, but its value depends on the regularization procedure (for a detailed discussion of this uncertainty, see Ref. 23).

Although this derivation cannot give us a correct numerical factor, it opens a new way to a qualitative understanding of more complicated situations. For example, the minimal conductivity of order of $e^{2} / h$ per channel has been observed experimentally also for the bilayer graphene $\stackrel{43}{ }$ with the energy spectrum drastically different from that for the singlelayer case. The bilayer graphene is a zero-gap semiconductor with parabolic touching of the electron and hole bands described by the single-particle Hamiltonian $\underline{\underline{43}, \underline{4}}$

$$
H_{\mathbf{p}}=\left(\begin{array}{cc}
0 & \left(p_{x}-i p_{y}\right)^{2} / 2 m \\
\left(p_{x}+i p_{y}\right)^{2} / 2 m & 0
\end{array}\right)
$$

(here we ignore some complications due to large-scale hopping processes which are important for a very narrow range of the Fermi energies ${ }^{44}$ ). The Hamiltonian (11) can be diagonalized by the unitary transformation $U_{\mathbf{p}}$ with the replacement $\phi_{\mathbf{p}} \rightarrow 2 \phi_{\mathbf{p}}$. Thus, the current operator after the transformation takes the form (6) with the replacement $v \rightarrow p / m, e^{-i \phi_{\mathbf{p}}} \rightarrow e^{-2 i \phi_{\mathbf{p}}}$. In contrast with the single-layer case, the density of electron states for the Hamiltonian (11) is finite at zero energy but the square of the current is, vice versa, linear in energy. As a result, we have the same estimate (10).

The Zitterbewegung processes play an important role when deriving quantum kinetic equation for electrons in graphene taking into account a static disorder ${ }^{34}$. For undoped case $\mu=0$ the electron and hole states are degenerate in energy, and interband (Zitterbewegung) scattering processes are similar, in a sense, to spin-flip scattering processes in the Kondo problem ${ }^{45}$. As was shown in Ref. 34 for a particular case of short-range scatterers, the Zitterbewegung processes lead to corrections to the static conductivity which are logarithmically divergent, and an exponentially small energy scale similar to the Kondo temperature arises

$$
\epsilon_{\mathrm{K}}=\epsilon_{c} e^{-\pi \sigma_{\mathrm{B}}}
$$

where $\epsilon_{c}$ the a cutoff energy of order of a bandwidth and $\sigma_{B}$ is the conventional Boltzmann conductivity in the units of $e^{2} / h$. Earlier this energy scale appeared in nonlinear sigma model approach to the conductivity of two-dimensional Dirac fermions ${ }^{13,14}$. This makes the classical Boltzmann equation inapplicable for a very small doping where $|\mu|<\epsilon_{\mathrm{K}}$.

\section{LANDAUER FORMULA APPROACH}

A deeper understanding of the origin of finite conductivity without charge carriers can be reached using Landauer formula approach ${ }^{25}$. Following Ref. 21 we consider the simplest possible geometry, choosing the sample as a ring of length $L_{y}$ in $y$ direction; we will use Landauer formula to calculate the conductance in $x$ direction (see Fig. 1). As we will see, the conductivity turns out to be dependent on the shape of the sample. To have a final transparency we should keep $L_{x}$ finite. On the other hand, periodic boundary conditions in $y$ direction are nonphysical and we have to choose $L_{y}$ as large as possible to weaken their effects. Thus, for two-dimensional situation one should choose $L_{x} \ll L_{y}$. In Ref. 22 the boundary conditions has been chosen in two different ways, with metallic armchair edges, and with infinite gap opening at the edges. The results for the conductivity in the limit $L_{x} \ll L_{y}$ turned out to be independent on the boundary conditions.

In the coordinate representation the Dirac equation at zero energy takes the form

$$
\begin{aligned}
& \left(K_{x}+i K_{y}\right) \psi_{1}=0 \\
& \left(K_{x}-i K_{y}\right) \psi_{2}=0
\end{aligned}
$$




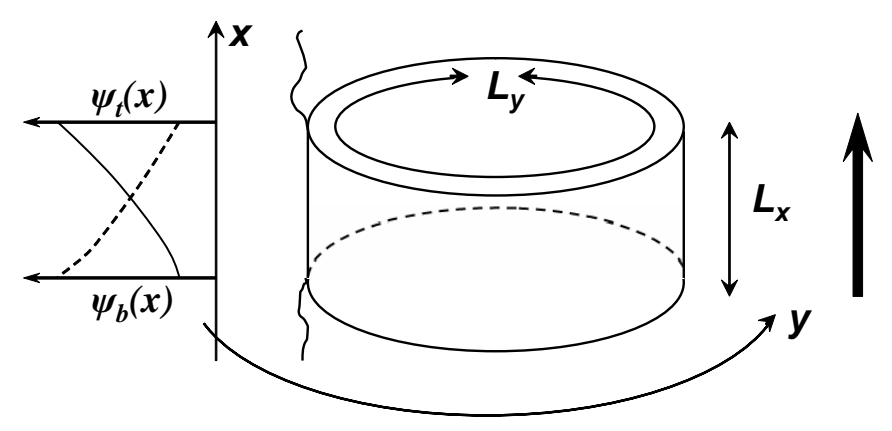

FIG. 1: Geometry of the sample. Thick arrow shows the direction of current. $\psi_{t}$ (solid line) and $\psi_{b}$ (dashed line) are wave functions of the edge states localized near the top and the bottom of the sample, correspondingly.

where $K_{i}=-i \frac{\partial}{\partial x_{i}}$. General solutions of these equations are just arbitrary analytical (or complex conjugated analytical) functions:

$$
\begin{aligned}
& \psi_{1}=\psi_{1}(x+i y), \\
& \psi_{2}=\psi_{2}(x-i y)
\end{aligned}
$$

Due to periodicity in $y$ direction both wave functions should be proportional to $\exp \left(i k_{y} y\right)$ where $k_{y}=2 \pi n / L_{y}, n=$ $0, \pm 1, \pm 2, \ldots$. This means that the dependence on $x$ is also fixed: the wave functions are proportional to $\exp \left( \pm 2 \pi n x / L_{y}\right)$. They correspond to the states localized near the bottom and top of the sample (see Fig. 1).

To use the Landauer formula, we should introduce boundary conditions at the sample edges $\left(x=0\right.$ and $\left.x=L_{x}\right)$. To be specific, let us assume that the leads are made of doped graphene with the potential $V_{0}<0$ and the Fermi energy $E_{F}=v k_{F}=-V_{0}$. The wave functions in the leads are supposed to have the same $y$-dependence, that is, $\psi_{1,2}(x, y)=\psi_{t, b}(x) \exp \left(i k_{y} y\right)$. Thus, one can try the solution of the Dirac equation in the following form:

$$
\begin{aligned}
& \psi_{t}(x)=\left\{\begin{array}{cc}
e^{i k_{x} x}+r e^{-i k_{x} x}, & x<0 \\
a e^{k_{y} x}, & 0<x<L_{x} \\
t e^{i k_{x} x}, & x>L_{x}
\end{array}\right. \\
& \psi_{b}(x)=\left\{\begin{array}{cc}
e^{i k_{x} x+i \phi}-r e^{-i k_{x} x-i \phi}, & x<0 \\
b e^{-k_{y} x}, & 0<x<L_{x} \\
t e^{i k_{x} x+i \phi}, & x>L_{x}
\end{array}\right.
\end{aligned}
$$

where $\sin \phi=k_{y} / k_{F}, k_{x}=\sqrt{k_{F}^{2}-k_{y}^{2}}$. From the conditions of continuity of the wave functions, one can find the transmission coefficient

$$
T_{n}=\left|t\left(k_{y}\right)\right|^{2}=\frac{\cos ^{2} \phi}{\cosh ^{2}\left(k_{y} L_{x}\right)-\sin ^{2} \phi} .
$$

Further, one should assume that $k_{F} L_{x} \gg 1$ and put $\phi \simeq 0$ in Eq.(16), so,

$$
T_{n}=\frac{1}{\cosh ^{2}\left(k_{y} L_{x}\right)}
$$

The conductance $G$ (per spin per valley $)^{25}$ and Fano factor of the shot noise ${ }^{26} F$ are expressed via the transmission coefficients (17) as

$$
\begin{aligned}
& G=\frac{e^{2}}{h} \sum_{n=-\infty}^{\infty} T_{n} \\
& F=1-\frac{\sum_{n=-\infty}^{\infty} T_{n}^{2}}{\sum_{n=-\infty}^{\infty} T_{n}}
\end{aligned}
$$


Note that in the ballistic regime where transmission probability for a given channel is either one or zero, $F=0$ (the current is noiseless) whereas if all $T_{n} \ll 1$ (e.g., current through tunnel junctions) $F \approx 1$.

Thus, the trace of the transmission coefficient which is just the conductance (in units of $e^{2} / h$ ) is

$$
\operatorname{Tr} T=\sum_{n=-\infty}^{\infty} \frac{1}{\cosh ^{2}\left(k_{y} L_{x}\right)} \simeq \frac{L_{y}}{\pi L_{x}}
$$

Assuming that the conductance is equal to $\sigma \frac{L_{y}}{L_{x}}$ one finds the contribution to the conductivity equal to $e^{2} /(\pi h)^{21,22}$. Note also that for the case of nanotubes $\left(L_{x} \gg L_{y}\right)$ one has a conductance $e^{2} / h$ per channel, in accordance with known results $46,47$.

For the Fano factor one has $F=1 / 3$ (Ref. 22). This result is very far from the ballistic regime and coincides with that for strongly disordered metals $\underline{48,49}$. This means that, in a sense, the Zitterbewegung works as an intrinsic disorder.

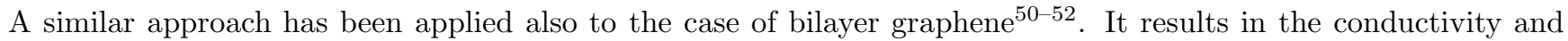
Fano factor of the same order as those for the single layer. However, in contrast with the latter case where the results are robust, for the bilayer numerical factors are dependent on the order or limits (chemical potential to zero, ratio of the contact layer thickness to the sample size, etc. $\stackrel{50,51}{\text {, }}$, and are sensitive to the trigonal warping 52 , small deviations from parabolic electron spectrum in the bilayer graphene $\underline{44}$.

\section{CONFORMAL MAPPING}

Thus, electron transport in undoped graphene is due to zero modes of the Dirac operator which are represented by analytic functions of $z=x+i y$ determined by boundary conditions. For the geometry shown in Fig. 1 these functions are just exponents:

$$
\psi_{1 n}(z)=\exp \left(2 \pi n z / L_{y}\right)
$$

so, a generic wave function inside the graphene flake can be written as

$$
\Psi(x, y) \equiv \sum_{n=-\infty}^{\infty} a_{n}\left(\begin{array}{c}
e^{2 \pi n z / L_{y}} \\
0
\end{array}\right)+b_{n}\left(\begin{array}{c}
0 \\
e^{2 \pi n z^{\star} / L_{y}}
\end{array}\right)
$$

where the coefficients $a_{n}, b_{n}$ are determined by the boundary conditions. Let the Fermi wavelength in the leads much smaller than geometric sizes of the flake. Then, for the most of modes one can write the boundary conditions assuming normal incidence, $\phi=0$ :

$$
\begin{aligned}
\psi_{\text {in }} & \equiv\left(\begin{array}{l}
1+r \\
1-r
\end{array}\right) \\
\psi_{\text {out }} & \equiv\left(\begin{array}{l}
t \\
t
\end{array}\right)
\end{aligned}
$$

where subscripts "in" and "out" label the values of the wave functions at the boundaries between the leads and the sample. In this approximation it is very easy to solve the problem of electron transport through graphene quantum dot of arbitrary shape using a conformal mapping of this shape to the stripe 28,29 . For example, the mapping

$$
w(z)=R_{1} e^{2 \pi i z / L_{y}}
$$

with $e^{2 \pi L_{y} / L_{x}}=R_{2} / R_{1}$ transforms the rectangular stripe $L_{x} \times L_{y}$ into the circular ring with inner and outer radii $R_{1}$ and $R_{2}$, respectively. Instead of Eq.(21) one can try in this case the solutions as

$$
\Psi(x, y) \equiv \sum_{n=-\infty}^{\infty} a_{n}\left(\begin{array}{c}
z^{n} \\
0
\end{array}\right)+b_{n}\left(\begin{array}{c}
0 \\
\left(z^{\star}\right)^{n}
\end{array}\right)
$$

The conformal mapping allows us to find immediately the solution for Corbino geometry where in and out leads are attached to inner and outer edges of the ring, respectively. Moreover, the solution of the problem for any shape of the flake topologically equivalent of the ring can be written automatically in terms of the corresponding conformal mapping 29 . Earlier $\underline{28}$ this method was applied to the case of graphene quantum dots with thin leads attached. Following Ref. 31 we will present in the next section the results for the Corbino geometry in a more general case, in the presence of magnetic fields. 


\section{AHARONOV-BOHM EFFECT IN UNDOPED GRAPHENE}

The Aharonov-Bohm (AB) effect ${ }^{53,54}$ is the shift of interference patterns from different electron trajectories by the magnetic flux through the area between the trajectories. This leads to oscillations of observable quantities such as conductance as a function of the magnetic flux. The AB effect in graphene has been studied already theoretically ${ }^{55-57}$ and experimentally ${ }^{58,59}$ for the case of a finite doping. It is not clear a priori whether this effect is observable or not

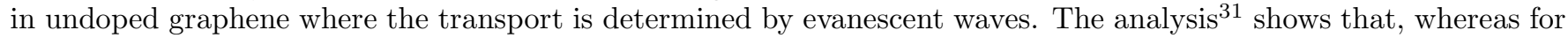
the case of very thin rings the $\mathrm{AB}$ oscillations are exponentially small, for a reasonable ratio of radii, such as, $R_{2} / R_{1}$ $=5$, the effect is quite observable.

Combining the conformal mapping technique with a general consideration of zero-energy states for massless Dirac fermions 60 one can derive simple and general rigorous formulas for any graphene flake topologically equivalent to the ring, avoiding both numerical simulations and explicit solutions of Shrödinger equation for some particular cases. Note that for the case of circular ring and constant magnetic field the problem can be solved exactly for any doping 61 but, of course, the mathematics required is much more cumbersome. In the corresponding limits, the results are, of course, the same.

For the case of zero energy $E=0$ (undoped graphene) the Dirac equation

$$
\sigma(-i \nabla-\mathbf{A}) \psi=E \psi
$$

for the two-component spinor $\psi, \mathbf{A}$ is the vector potential and we use the units $\hbar=e=1$, splits into two independent equations for the components $\psi_{\sigma}(\sigma= \pm)$ :

$$
\left(-i \frac{\partial}{\partial x}+\sigma \frac{\partial}{\partial y}-A_{x}-i \sigma A_{y}\right) \psi_{\sigma}=0
$$

We will use the gauge $\nabla \mathbf{A}=0$ and introduce scalar magnetic potential $\varphi$ by

$$
A_{x}=\frac{\partial \varphi}{\partial y}, A_{y}=-\frac{\partial \varphi}{\partial x}
$$

thus,

$$
\nabla^{2} \varphi=-B
$$

where $B$ is the magnetic induction. The vector potential can be eliminated by a substitution 60

$$
\psi_{\sigma}=e^{\sigma \varphi} \chi_{\sigma}
$$

Eq.(26) being satisfied for an arbitrary analytic function $\chi_{+}(z)$ and complex conjugated analytic function $\chi_{-}(\bar{z})$. The latter can be found from boundary conditions as described in the previous section. Thus, the boundary conditions following from Eq.(22) are

$$
\begin{aligned}
1+r & =\psi_{+}^{(1)} \\
1-r & =\psi_{-}^{(1)} \\
t & =\psi_{+}^{(2)} \\
t & =\psi_{-}^{(2)}
\end{aligned}
$$

where superscripts 1 and 2 label the boundaries attached to the corresponding leads.

If the boundary of the sample is simply connected one can always choose $\varphi=0$ at the boundary and, thus, the magnetic field disappears from Eq.(30); this fact was used in Ref. 30 as a very elegant way to prove that a random vector potential does not change the value of minimal conductivity. Further we will consider a sample with a topology of the ring where the scalar potential $\varphi$ is still constant at each boundary but these constants, $\varphi_{1}$ and $\varphi_{2}$ are different. Also, by symmetry,

$$
\chi_{+}^{(2)} / \chi_{+}^{(1)}=\chi_{-}^{(1)} / \chi_{-}^{(2)}
$$

The answer for the transmission coefficient $T=|t|^{2}$ has the form:

$$
T_{j}=\frac{1}{\cosh ^{2}[2(j+a) \ln \Lambda]}
$$


where $j= \pm 1 / 2, \pm 3 / 2, \ldots$ labels zero modes of the Dirac equation, $\Lambda$ is determined by a conformal transformation of our flake to the rectangle and

$$
a=\frac{\varphi_{2}-\varphi_{1}}{2 \ln \Lambda}
$$

which generalizes the corresponding result of Ref. 29 on the case of finite magnetic fields.

For simplicity, we will consider further the case of the Corbino disc with inner radius $R_{1}$ and outer radius $R_{2}$, when 29

$$
\Lambda=\sqrt{R_{2} / R_{1}}
$$

The conductance $G$ (per spin per valley) and Fano factor of the shot noise $F$ are expressed via the transmission coefficients (32) via Eq.(18). To calculate the sums one can use the Poisson summation formula

$$
\sum_{n=-\infty}^{\infty} f(n+x)=\sum_{k=-\infty}^{\infty} \exp [-2 i \pi k x] \widetilde{f}_{k}
$$

where $n, k$ are all integers and $\widetilde{f}_{k}$ is the Fourier transform of the function $f(x)$. Substituting Eq.(32) into (18) one finds a compact and general answer for the effect of magnetic field on the transport characteristics:

$$
\begin{gathered}
G=\frac{e^{2}}{h \ln \Lambda}\left[1+2 \sum_{k=1}^{\infty}(-1)^{k} \cos (2 \pi k a) \alpha_{k}\right] \\
F=1-\frac{2}{3}\left[\frac{1+2 \sum_{k=1}^{\infty}(-1)^{k} \cos (2 \pi k a) \alpha_{k}\left(1+\frac{\pi^{2} k^{2}}{4 \ln ^{2} \Lambda}\right)}{1+2 \sum_{k=1}^{\infty}(-1)^{k} \cos (2 \pi k a) \alpha_{k}}\right]
\end{gathered}
$$

where

$$
\alpha_{k}=\frac{\pi^{2} k / 2 \ln \Lambda}{\sinh \left(\pi^{2} k / 2 \ln \Lambda\right)}
$$

Equation (28) can be solved explicitly for radially symmetric distributions of the magnetic field $B(r)$ :

$$
\varphi_{2}-\varphi_{1}=\frac{\Phi}{2 \pi} \ln \left(\frac{R_{2}}{R_{1}}\right)+\int_{R_{1}}^{R_{2}} \frac{d r}{r} \int_{R_{1}}^{r} d r^{\prime} r^{\prime} B\left(r^{\prime}\right)
$$

where $\Phi$ is the magnetic flux through the inner ring. In the case of AB effect where all magnetic flux is concentrated within the inner ring one has

$$
a=\frac{e \Phi}{2 \pi \hbar c}
$$

where we have restored world constants.

Due to the large factor $\pi^{2}$ in the argument of $\sinh$ in Eq.(38) only the terms with $k=1$ should be kept in Eqs. (36) and (37) for all realistic shapes, thus,

$$
\begin{gathered}
G=G_{0}\left[1-\frac{4 \pi^{2}}{\ln \left(R_{2} / R_{1}\right)} \exp \left(-\frac{\pi^{2}}{\ln \left(R_{2} / R_{1}\right)}\right) \cos \left(\frac{e \Phi}{\hbar c}\right)\right] \\
F=\frac{1}{3}+\frac{8 \pi^{4}}{3 \ln ^{3}\left(R_{2} / R_{1}\right)} \exp \left(-\frac{\pi^{2}}{\ln \left(R_{2} / R_{1}\right)}\right) \cos \left(\frac{e \Phi}{\hbar c}\right)
\end{gathered}
$$

where

$$
G_{0}=\frac{2 e^{2}}{h \ln \left(R_{2} / R_{1}\right)}
$$


is the conductance of the ring without magnetic field ${ }^{29}$.

Oscillating contributions to $G$ and $F$ are exponentially small for very thin rings but, for sure, measurable if the ring is thick enough. For $R_{2} / R_{1}=5$ their amplitudes are $5.3 \%$ and $40 \%$, respectively.

Consider now a generic case with the magnetic field $B=0$ within the flake. Then, the solution of Eq.(28) is a harmonic function, that is, real or imaginary part by an analytic function. It can be obtained from the solution for the disc by the same conformal transformation which determines the function $\Lambda$. One can see immediately that Eq. (40) remains the same. As for the expressions (41), (42) they can be rewritten in terms of experimentally measurable quantity $G_{0}$,

$$
\begin{gathered}
G=G_{0}\left[1-\frac{4 \pi^{2}}{\beta} \exp \left(-\pi^{2} / \beta\right) \cos \left(\frac{e \Phi}{\hbar c}\right)\right] \\
F=\frac{1}{3}+\frac{8 \pi^{4}}{3 \beta^{3}} \exp \left(-\pi^{2} / \beta\right) \cos \left(\frac{e \Phi}{\hbar c}\right)
\end{gathered}
$$

where $\beta=2 e^{2} / h G_{0}$ and we assume $\beta \ll \pi^{2}$.

Thus, conformal transformations $\frac{28,29}{}$ is a powerful tool to consider pseudodiffusive transport in undoped graphene flakes of arbitrary shape, not only without magnetic field but also in the presence of magnetic fluxes in the system. Experimental study of the Aharonov-Bohm oscillations and comparison with simple expressions (44), (45) derived here would be a suitable way to check whether the ballistic (pseudodiffusive) regime is reached or not in a given experimental situation.

\section{CONCLUSIONS}

Undoped graphene is a gapless semiconductor, with filled valence band and empty conduction band. It is really counterintuitive that in such a situation, at zero temperature, it has a finite conductivity, of order of the conductance quantum $e^{2} / h$. This is one of the most striking consequences of its peculiar "ultrarelativistic" energy spectrum. More precisely, this is the consequence of chirality of electron states. In the case of bilayer ${ }^{43}$ the electron energy spectrum is parabolic but the states are chiral, with the Berry phase $2 \pi$, and this leads to the finite minimal conductivity $50-52$ as discussed above.

Formally, the electron transport in undoped graphene is determined by zero modes of the Dirac operator which are described by analytic functions with proper boundary conditions. Therefore, the whole power of complex calculus can be used here, as well as in classical old-fashionable branches of mathematical physics such as two-dimensional hydrodynamics or electrostatics. These states cannot correspond to the waves propagating through the sample but, rather, are represented by evanescent waves. The transport via evanescent waves in undoped graphene is a completely new variety of electron transport in solids, drastically different from all types known before (ballistic transport in nanowires and constrictions, diffusive transport in dirty metals, variable-range-hopping transport in Anderson insulators, etc.). Deeper understanding of this new quantum phenomena seems to be a very important problem.

The work is financially supported by Stichting voor Fundamenteel Onderzoek der Materie (FOM), the Netherlands.

1 A. K. Geim and K. S. Novoselov, Nature Mater. 6,183 (2007).

2 M. I. Katsnelson, Mater. Today 10, 20 (2007).

3 C. W. J. Beenakker, Rev. Mod. Phys. 80, 1337 (2008).

4 A. H. Castro Neto, F. Guinea, N. M. R. Peres, K. S. Novoselov, and A. K. Geim, Rev. Mod. Phys. 80, 315 (2008).

5 A. K. Geim, Science 324, 1530 (2009).

6 S. Das Sarma, S. Adam, E. H. Hwang, and E. Rossi, arXiv:1003.4731

7 M. A. H. Vozmediano, M. I. Katsnelson, and F. Guinea, Phys. Rep. 496, 109 (2010).

8 K. S. Novoselov, A. K. Geim, S. V. Morozov, D. Jiang, M. I. Katsnelson, I. V. Grigorieva, S. V. Dubonos, and A. A. Firsov, Nature 438, 197 (2005).

9 Y. Zhang, Y.-W. Tan, H. L. Stormer, and P. Kim, Nature 438, 201 (2005).

10 M. I. Katsnelson, K. S. Novoselov, and A. K. Geim, Nature Phys. 2, 620 (2006).

11 N. Stander, B. Huard, and D. Goldhaber-Gordon, Phys. Rev. Lett. 102, 026807 (2009).

12 A. F. Young and P. Kim, Nature Phys. 5, 222 (2009).

13 E. Fradkin, Phys. Rev. B 33, 3263 (1986).

14 P. A. Lee, Phys. Rev. Lett. 71, 1887 (1993). 
15 A. W. W. Ludwig, M. P. A. Fisher, R. Shankar, and G. Grinstein, Phys. Rev. B 50, 7526 (1994).

16 A. A. Nersesyan, A. M. Tsvelik, and F. Wenger, Phys. Rev. Lett. 72, 2628 (1994).

17 K. Ziegler, Phys. Rev. Lett. 80, 3113 (1998).

18 N. H. Shon and T. Ando, J. Phys. Soc. Japan 67, 2421 (1998).

19 E. V. Gorbar, V. P. Gusynin, V. A. Miransky, and I. A. Shovkovy, Phys. Rev. B 66, 045108 (2002).

20 X. Yang and C. Nayak, Phys. Rev. B 65, 064523 (2002).

21 M. I. Katsnelson, Eur. Phys. J. B 51, 157 (2006).

22 J. Tworzydlo, B. Trauzettel, M. Titov, A. Rycerz, and C. W. J. Beenakker, Phys. Rev. Lett. 96, 246802 (2006).

23 S. Ryu, C. Mudry, A. Furusaki, and A. W. W. Ludwig, Phys. Rev. B 75, 205344 (2007).

24 E. Schrödinger, Sitz. Preuss. Akad. Wiss. Phys.-Math. 24, 418 (1930).

25 C. W. J. Beenakker and H. van Houten, Solid State Phys. 44, 1 (1991).

26 Ya. M. Blanter and M. Büttiker, Phys. Rep. 336, 1 (2000).

27 E. Prada, P. San-Jose, B. Wunsch, and F. Guinea, Phys. Rev. B 75, 113407 (2007).

28 M. I. Katsnelson and F. Guinea, Phys. Rev. B, 78, 075417 (2008).

29 A. Rycerz, P. Recher, and M. Wimmer, Phys. Rev. B 80125417 (2009).

30 A. Schuessler, P. M. Ostrovsky, I. V. Gornyi, and A. D. Mirlin, Phys. Rev. B 79, 075405 (2009).

31 M. I. Katsnelson, Europhys. Lett. 89, 17001 (2010).

32 F. Miao, S. Wijeratne, Y. Zhang, U. C. Coskun, W. Bao, and C. N. Lau, Science 317, 1530 (2007).

33 R. Danneau, F. Wu, M. F.Craciun, S. Russo, M. Y. Tomi, J. Salmilehto, A. F. Morpurgo, and P. J. Hakonen, Phys. Rev. Lett. 100, 196802 (2008).

34 M. Auslender and M. I. Katsnelson, Phys. Rev. B 76, 235425 (2007).

35 R. Gerritsma, G. Kirchmair, F. Zähringer, E. Solano, R. Blatt, and C. F. Roos, Nature 463, 68 (2010).

36 J. Cserti and G. Dávid, Phys. Rev. B 74, 172305 (2006).

37 T. M. Rusin and W. Zawadzki, Phys. Rev. B 78, 125419 (2008).

38 T. M. Rusin and W. Zawadzki, Phys. Rev. B 80, 045416 (2009).

39 A. A. Abrikosov, Phys. Rev. B 58, 2788 (1998).

40 V. B. Berestetskii, E. M. Lifshitz, and L. P. Pitaevskii, Relativistic Quantum Theory, vol. 1 (Pergamon, Oxford etc., 1971).

41 A. S. Davydov, Quantum Mechanics (Pergamon, Oxford etc., 1976).

42 D. N. Zubarev, Nonequilibrium Statistical Thermodynamics (Consultants Bureau, New York, 1974).

43 K. S. Novoselov, E. McCann, S. V. Morozov, V. I. Falko, M. I. Katsnelson, U. Zeitler, D. Jiang, F. Schedin, and A. K. Geim, Nature Phys. 2, 177 (2006).

44 E. McCann and V. I. Falko, Phys. Rev. Lett. 96, 086805 (2006).

45 A. C. Hewson, The Kondo Problem to Heavy Fermions (Cambridge University Press, Cambridge, 1993).

${ }^{46}$ W. Tian and S. Datta, Phys. Rev. B 49, 5097 (1994).

47 L. Chico, L. X. Benedict, S. G. Louie, and M. L. Cohen, Phys. Rev. B 54, 2600 (1996).

48 C. W. J. Beenakker and M. Büttiker, Phys. Rev. B 46, 1889 (1992).

49 K. E. Nagaev, Phys. Lett. A 169, 103 (1992).

50 M. I. Katsnelson, Eur. Phys. J. B 52, 151 (2006).

51 I. Snyman and C. W. J. Beenakker, Phys. Rev. B 75, 045322 (2007).

52 J. Cserti, A. Csordás, and G. Dávid, Phys. Rev. Lett. 99, 066802 (2007).

${ }^{53}$ Y. Aharonov and D. Bohm, Phys. Rev. 115, 485 (1959).

54 S. Olariu andI. I. Popescu, Rev. Mod. Phys. 57, 339 (1985).

55 P. Recher, B. Trauzettel, A. Rycerz, Ya. M. Blanter, C. W. J. Beenakker, and A. F. Morpurgo, Phys. Rev. B 76, 235404 (2007).

56 R. Jackiw, A. I. Milstein, S.-Y. Pi, and I. S. Terekhov, Phys. Rev. B 80, 033413 (2009).

57 J. Wurm, M. Wimmer, H. U. Baranger, and K. Richter, Semicond. Sci. Tech. 25, 034003 (2010).

58 S. Russo, J. B. Oostinga, D. Wehenkel, H. B. Heersche, S. S. Sobhani, L. M. K. Vandersypen, and A. F. Morpurgo, Phys. Rev. B 77, 085413 (2008).

59 M. Huefner, F. Molitor, A. Jacobsen, A. Pioda, C. Stampfer, K. Ensslin, and T. Ihn, Phys. Stat. Sol. (b) 246, 2756 (2009).

60 Y. Aharonov and A. Casher, Phys. Rev. A 19, 2461 (1979).

61 A. Rycerz, Phys. Rev. B 81, 121404 (2010). 